\title{
SIMULTANEOUS LINEARIZATION OF HOLOMORPHIC GERMS IN PRESENCE OF RESONANCES
}

\author{
JASMIN RAISSY
}

\begin{abstract}
Let $f_{1}, \ldots, f_{m}$ be $m \geq 2$ germs of biholomorphisms of $\mathbb{C}^{n}$, fixing the origin, with $\left(\mathrm{d} f_{1}\right)_{O}$ diagonalizable and such that $f_{1}$ commutes with $f_{h}$ for any $h=2, \ldots, m$. We prove that, under certain arithmetic conditions on the eigenvalues of $\left(\mathrm{d} f_{1}\right)_{O}$ and some restrictions on their resonances, $f_{1}, \ldots, f_{m}$ are simultaneously holomorphically linearizable if and only if there exists a particular complex manifold invariant under $f_{1}, \ldots, f_{m}$.
\end{abstract}

\section{INTRODUCTION}

One of the main questions in the study of local holomorphic dynamics (see A and $[\mathrm{B}$ for general surveys on this topic) is when a given germ of biholomorphism $f$ of $\mathbb{C}^{n}$ at a fixed point $p$, which we may place at the origin $O$, is holomorphically linearizable, i.e., there exists a local holomorphic change of coordinates, tangent to the identity, conjugating $f$ to its linear part. The answer to this question depends on the set of eigenvalues of $\mathrm{d} f_{O}$, usually called the spectrum of $\mathrm{d} f_{O}$. In fact, if we denote by $\lambda_{1}, \ldots, \lambda_{n} \in \mathbb{C}^{*}$ the eigenvalues of $\mathrm{d} f_{O}$, then it may happen that there exists a multi-index $k=\left(k_{1}, \ldots, k_{n}\right) \in \mathbb{N}^{n}$ with $|k|:=k_{1}+\cdots+k_{n} \geq 2$ and such that

$$
\lambda^{k}-\lambda_{j}:=\lambda_{1}^{k_{1}} \cdots \lambda_{n}^{k_{n}}-\lambda_{j}=0
$$

for some $1 \leq j \leq n$; a relation of this kind is called a resonance of $f$, and $k$ is called a resonant multi-index. A resonant monomial is a monomial $z^{k}=z_{1}^{k_{1}} \cdots z_{n}^{k_{n}}$ in the $j$-th coordinate such that $\lambda^{k}=\lambda_{j}$.

One possible generalization of the previous question is to ask when a given set of $m \geq 2$ germs of biholomorphisms $f_{1}, \ldots, f_{m}$ of $\mathbb{C}^{n}$ at the same fixed point, which we may place at the origin, are simultaneously holomorphically linearizable, i.e., there exists a local holomorphic change of coordinates conjugating $f_{h}$ to its linear part for each $h=1, \ldots, m$.

In $[\mathrm{R}]$ we found, under certain arithmetic conditions on the eigenvalues and some restrictions on the resonances, a necessary and sufficient condition for holomorphic linearization. In this article we shall use that result to find a necessary and sufficient condition for holomorphic simultaneous linearization.

Received by the editors February 13, 2009 and, in revised form, July 27, 2009.

2010 Mathematics Subject Classification. Primary 37F50; Secondary 32H50.

Key words and phrases. Linearization problem, commuting holomorphic maps, resonances, small divisors, Brjuno condition.

(C)2009 American Mathematical Society Reverts to public domain 28 years from publication 
Before stating our result we need the following definitions:

Definition 1.1. Let $1 \leq s \leq n$. We say that $\boldsymbol{\lambda}=\left(\lambda_{1}, \ldots, \lambda_{s}, \mu_{1}, \ldots, \mu_{r}\right) \in\left(\mathbb{C}^{*}\right)^{n}$ has only level $s$ resonances if there are only two kinds of resonances:

$$
\lambda^{k}=\lambda_{h} \Longleftrightarrow k \in \widetilde{K}_{1} \text {, }
$$

where

$$
\widetilde{K}_{1}=\left\{k \in \mathbb{N}^{n}|| k \mid \geq 2, \sum_{p=1}^{s} k_{p}=1 \text { and } \mu_{1}^{k_{s+1}} \cdots \mu_{r}^{k_{n}}=1\right\}
$$

and

$$
\lambda^{k}=\mu_{j} \Longleftrightarrow k \in \widetilde{K}_{2}
$$

where

$\widetilde{K}_{2}=\left\{k \in \mathbb{N}^{n}|| k \mid \geq 2, k_{1}=\cdots=k_{s}=0\right.$ and $\exists j \in\{1, \ldots, r\}$ s.t. $\left.\mu_{1}^{k_{s+1}} \cdots \mu_{r}^{k_{n}}=\mu_{j}\right\}$.

For $s=n$ having only level $s$ resonances means that there are no resonances. When $s<n$, if $\left(\lambda_{1}, \ldots, \lambda_{s}\right)$ have no resonances, it is not difficult to verify that $\boldsymbol{\lambda}=\left(\lambda_{1}, \ldots, \lambda_{s}, 1, \ldots, 1\right)$ has only level $s$ resonances.

Definition 1.2. Let $n \geq 2$ and let $\lambda_{1}, \ldots, \lambda_{n} \in \mathbb{C}^{*}$ be not necessarily distinct. For any $m \geq 2$, put

$$
\widetilde{\omega}(m)=\min _{\substack{2 \leq|k| \leq m \\ k \notin \operatorname{Res}_{j}(\lambda)}} \min _{1 \leq j \leq n}\left|\lambda^{k}-\lambda_{j}\right|,
$$

where $\operatorname{Res}_{j}(\lambda)$ is the set of multi-indices $k \in \mathbb{N}^{n}$, with $|k| \geq 2$, giving a resonance relation for $\lambda=\left(\lambda_{1}, \ldots, \lambda_{n}\right)$ relative to $1 \leq j \leq n$, i.e., such that $\lambda^{k}-\lambda_{j}=0$. We say that $\lambda$ satisfies the reduced Brjuno condition if there exists a strictly increasing sequence of integers $\left\{p_{\nu}\right\}_{\nu_{\geq} 0}$ with $p_{0}=1$ such that

$$
\sum_{\nu \geq 0} p_{\nu}^{-1} \log \widetilde{\omega}\left(p_{\nu+1}\right)^{-1}<\infty
$$

Note that the reduced Brjuno condition of order $n$ (i.e., when there are no resonances) is nothing but the usual Brjuno condition introduced in $\mathrm{Br}$. (see also [M], pp. 25-37, for the one-dimensional case).

Definition 1.3. Let $f$ be a germ of biholomorphism of $\mathbb{C}^{n}$ fixing the origin $O$ and let $s \in \mathbb{N}$, with $1 \leq s \leq n$. The origin $O$ is called a quasi-Brjuno fixed point of order $s$ if $\mathrm{d} f_{O}$ is diagonalizable and, denoting by $\boldsymbol{\lambda}$ the spectrum of $\mathrm{d} f_{O}$, we have:

(i) $\boldsymbol{\lambda}$ has only level $s$ resonances;

(ii) $\boldsymbol{\lambda}$ satisfies the reduced Brjuno condition.

We say that $f$ has the origin as a quasi-Brjuno fixed point if there exists $1 \leq s \leq n$ such that it is a quasi-Brjuno fixed point of order $s$.

Definition 1.4. Let $f_{1}, \ldots, f_{m}$ be $m$ germs of biholomorphisms of $\mathbb{C}^{n}$, fixing the origin, with $m \geq 2$, and let $M$ be a germ of complex manifold at $O$ of codimension $1 \leq s \leq n$, and $f_{h}$-invariant for each $h=1, \ldots, m$. We say that $M$ is a simultaneous osculating manifold for $f_{1}, \ldots, f_{m}$ if there exists a holomorphic flat $(1,0)$ connection $\nabla$ of the normal bundle $N_{M}$ of $M$ in $\mathbb{C}^{n}$ commuting with $\left.\mathrm{d} f_{h}\right|_{N_{M}}$ for each $h=1, \ldots, m$. 
In $[\mathrm{R}]$ we saw that the osculating condition was necessary and sufficient to extend a holomorphic linearization from an invariant submanifold to a whole neighbourhood of the origin for a germ $f_{1}$ of biholomorphism with a quasi-Brjuno fixed point. Our main theorem shows that the simultaneous osculating condition is also necessary and sufficient to extend a common holomorphic linearization, just assuming that $f_{1}$ has a quasi-Brjuno fixed point and commutes with $f_{2}, \ldots, f_{m}$ :

Theorem 1.1. Let $f_{1}, \ldots, f_{m}$ be $m \geq 2$ germs of biholomorphisms of $\mathbb{C}^{n}$, fixing the origin. Assume that $f_{1}$ has the origin as a quasi-Brjuno fixed point of order $s$, with $1 \leq s \leq n$, and that it commutes with $f_{h}$ for any $h=2, \ldots, m$. Then $f_{1}, \ldots, f_{m}$ are simultaneously holomorphically linearizable if and only if there exists a germ of complex manifold $M$ at $O$ of codimension s, invariant under $f_{h}$ for each $h=1, \ldots, m$, which is a simultaneous osculating manifold for $f_{1}, \ldots, f_{m}$ and such that $\left.f_{1}\right|_{M}, \ldots,\left.f_{m}\right|_{M}$ are simultaneously holomorphically linearizable.

A similar topic is studied in $[\mathrm{S}$. However, his results are not comparable with ours, because his notion of "linearization modulo an ideal" is not suitable for producing a full linearization result, except when there are no resonances at all, whereas in our result we explicitly admit some resonances.

We shall need the following notation: if $g: \mathbb{C}^{n} \rightarrow \mathbb{C}$ is a holomorphic function with $g(O)=0$, and $z=(x, y) \in \mathbb{C}^{n}$ with $x \in \mathbb{C}^{s}$ and $y \in \mathbb{C}^{n-s}$, we shall denote by $\operatorname{ord}_{x}(g)$ the maximum positive integer $m$ such that $g$ belongs to the ideal $\left\langle x_{1}, \cdots, x_{s}\right\rangle^{m}$. Furthermore, we shall say that the local coordinates $z=(x, y)$ are adapted to the complex submanifold $M$ if in those coordinates $M$ is given by $\{x=0\}$.

\section{LINEARIZATION}

We first introduced osculating manifolds in R. A germ $f$ of biholomorphism of $\mathbb{C}^{n}$ fixing the origin $O$ admits an osculating manifold $M$ of codimension $1 \leq s \leq n$ if there is a germ of $f$-invariant complex manifold $M$ at $O$ of codimension $s$ such that the normal bundle $N_{M}$ of $M$ admits a holomorphic flat $(1,0)$-connection that commutes with $\left.\mathrm{d} f\right|_{N_{M}}$. Definition 1.4 is the natural extension of this object to the case we are dealing with.

We shall need the following characterization of simultaneous osculating manifolds.

Proposition 2.1. Let $f_{1}, \ldots, f_{m}$ be $m$ germs of biholomorphisms of $\mathbb{C}^{n}$, fixing the origin, with $m \geq 2$, and let $M$ be a germ of complex manifold at $O$ of codimension $1 \leq s \leq n$, and $f_{h}$-invariant for each $h=1, \ldots, m$. Then $M$ is a simultaneous osculating manifold for $f_{1}, \ldots, f_{m}$ if and only if there exist local holomorphic coordinates $z=(x, y)$ about $O$ adapted to $M$ in which $f_{h}$ has the form

$$
\begin{array}{rlrl}
x_{i}^{\prime} & =\sum_{p=1}^{s} a_{i, p}^{(h)} x_{p}+\widehat{f}_{i}^{(h)}(x, y) & & \text { for } i=1, \ldots, s, \\
y_{j}^{\prime} & =f_{j}^{(h)}(x, y) & \text { for } j=1, \ldots, r=n-s,
\end{array}
$$

with

$$
\operatorname{ord}_{x}\left(\widehat{f}_{i}^{(h)}\right) \geq 2,
$$

for any $i=1, \ldots, s$ and $h=1, \ldots, m$. 
Proof. If there exist local holomorphic coordinates $z=(x, y)$ about $O$ adapted to $M$ in which $f_{h}$ has the form (2) with $\operatorname{ord}_{x}\left(\widehat{f}_{i}^{(h)}\right) \geq 2$ for any $i=1, \ldots, s$ and $h=1, \ldots, m$, then it is obvious to verify that the trivial holomorphic flat $(1,0)$ connection commutes with $\left.\mathrm{d} f_{h}\right|_{N_{M}}$ for each $h=1, \ldots, m$.

Conversely, let $\nabla$ be a holomorphic flat $(1,0)$-connection of the normal bundle $N_{M}$ commuting with $\left.\mathrm{d} f_{h}\right|_{N_{M}}$ for each $h=1, \ldots, m$. It suffices to choose local holomorphic coordinates $z=(x, y)$ adapted to $M$ in which all the connection coefficients $\Gamma_{j k}^{i}$ with respect to the local holomorphic frame $\left\{\pi\left(\frac{\partial}{\partial x_{1}}\right), \ldots, \pi\left(\frac{\partial}{\partial x_{s}}\right)\right\}$ of $N_{M}$ are zero (see $[\mathbf{R}$, Proposition 3.1 and Lemma 3.2), and then the assertion follows immediately from the proof of Theorem 1.3 of $[\mathrm{R}$.

Corollary 2.2. Let $f_{1}, \ldots, f_{m}$ be $m$ germs of biholomorphisms of $\mathbb{C}^{n}$, fixing the origin, with $m \geq 2$, and let $M$ be a germ of complex manifold at $O$ of codimension $1 \leq s \leq n$, and $f_{h}$-invariant for each $h=1, \ldots, m$. Then $M$ is a simultaneous osculating manifold for $f_{1}, \ldots, f_{m}$ such that $\left.f_{1}\right|_{M}, \ldots,\left.f_{m}\right|_{M}$ are simultaneously holomorphically linearizable if and only if there exist local holomorphic coordinates $z=(x, y)$ about $O$ adapted to $M$ in which $f_{h}$ has the form

$$
\begin{aligned}
x_{i}^{\prime} & =\sum_{p=1}^{s} a_{i, p}^{(h)} x_{h}+\widehat{f}_{i}^{(h), 1}(x, y) \quad \text { for } \quad i=1, \ldots, s, \\
y_{j}^{\prime} & =f_{j}^{(h) \operatorname{lin}}(x, y)+\widehat{f}_{j}^{(h), 2}(x, y) \quad \text { for } \quad j=1, \ldots, r=n-s,
\end{aligned}
$$

where $f_{j}^{(h) \operatorname{lin}}(x, y)$ is linear and

$$
\begin{aligned}
& \operatorname{ord}_{x}\left(\widehat{f}_{i}^{(h), 1}\right) \geq 2, \\
& \operatorname{ord}_{x}\left(\widehat{f}_{j}^{(h), 2}\right) \geq 1,
\end{aligned}
$$

for any $i=1, \ldots, s, j=1, \ldots, r$ and $h=1, \ldots, m$.

Proof. One direction is clear.

Conversely, thanks to Proposition 2.1, the fact that $M$ is a simultaneous osculating manifold for $f_{1}, \ldots, f_{m}$ is equivalent to the existence of local holomorphic coordinates $z=(x, y)$ about $O$ adapted to $M$, in which $f_{h}$ has the form (31) with $\operatorname{ord}_{x}\left(\widehat{f}_{i}^{(h), 1}\right) \geq 2$ for any $i=1, \ldots, s$ and $h=1, \ldots, m$. Furthermore, $\left.f_{1}\right|_{M}, \ldots,\left.f_{m}\right|_{M}$ are simultaneously holomorphically linearizable; therefore, there exists a local holomorphic change of coordinate, tangent to the identity, and of the form

$$
\begin{aligned}
& \tilde{x}=x, \\
& \tilde{y}=\Phi(y),
\end{aligned}
$$

conjugating $f_{h}$ to $\tilde{f}_{h}$ of the form (3) satisfying (4), for each $h=1, \ldots, m$, as we wanted.

Remark 2.3. It is possible to give the formal analogous of Definition 1.4, and then to prove a formal analogous of Proposition 2.1 and Corollary [2.2, exactly as in $[\mathrm{R}$.

In the proof of Theorem 1.1 we shall use the following result we proved in $[\mathrm{R}$. 
Theorem 2.4 (Raissy, 2007). Let $f$ be a germ of biholomorphism of $\mathbb{C}^{n}$ having the origin $O$ as a quasi-Brjuno fixed point of order s. Then $f$ is holomorphically linearizable if and only if it admits an osculating manifold $M$ of codimension s such that $\left.f\right|_{M}$ is holomorphically linearizable.

We can now prove our result.

Theorem 2.5. Let $f_{1}, \ldots, f_{m}$ be $m \geq 2$ germs of biholomorphisms of $\mathbb{C}^{n}$, fixing the origin. Assume that $f_{1}$ has the origin as a quasi-Brjuno fixed point of order $s$, with $1 \leq s \leq n$, and that it commutes with $f_{h}$ for any $h=2, \ldots, m$. Then $f_{1}, \ldots, f_{m}$ are simultaneously holomorphically linearizable if and only if there exists a germ of complex manifold $M$ at $O$ of codimension $s$, invariant under $f_{h}$ for each $h=1, \ldots, m$, which is a simultaneous osculating manifold for $f_{1}, \ldots, f_{m}$ and such that $\left.f_{1}\right|_{M}, \ldots,\left.f_{m}\right|_{M}$ are simultaneously holomorphically linearizable.

Proof. Let $M$ be a germ of complex manifold at $O$ of codimension $s$, invariant under $f_{h}$ for each $h=1, \ldots, m$ which is a simultaneous osculating manifold for $f_{1}, \ldots, f_{m}$ and such that $\left.f_{1}\right|_{M}, \ldots,\left.f_{m}\right|_{M}$ are simultaneously holomorphically linearizable. Thanks to the hypotheses we can choose local holomorphic coordinates

$$
(x, y)=\left(x_{1}, \ldots, x_{s}, y_{1}, \ldots, y_{r}\right)
$$

such that $f_{1}$ is of the form

$$
\begin{aligned}
& x_{i}^{\prime}=\lambda_{1, i} x_{i}+f_{i}^{(1), 1}(x, y) \quad \text { for } i=1, \ldots, s, \\
& y_{j}^{\prime}=\mu_{1, j} y_{j}+f_{j}^{(1), 2}(x, y) \quad \text { for } \quad j=1, \ldots, r=n-s,
\end{aligned}
$$

and, for $h=2, \ldots, m$, each $f_{h}$ is of the form

$$
\begin{array}{ll}
x_{i}^{\prime}=\sum_{p=1}^{s} a_{i, p}^{(h)} x_{p}+f_{i}^{(h), 1}(x, y) & \text { for } i=1, \ldots, s, \\
y_{j}^{\prime}=f_{j}^{(h) \operatorname{lin}}(x, y)+f_{j}^{(h), 2}(x, y) & \text { for } j=1, \ldots, r=n-s,
\end{array}
$$

where $f_{j}^{(h) \operatorname{lin}}(x, y)$ is linear, and for each $k=1, \ldots, m$,

$$
\begin{aligned}
& \operatorname{ord}_{x}\left(f_{i}^{(k), 1}\right) \geq 2, \\
& \operatorname{ord}_{x}\left(f_{j}^{(k), 2}\right) \geq 1,
\end{aligned}
$$

that is

$$
\begin{aligned}
f_{i}^{(k), 1}(x, y) & =\sum_{\substack{|K| \geq 2 \\
\left|K^{\prime}\right| \geq 2}} f_{K, i}^{(k), 1} x^{K^{\prime}} y^{K^{\prime \prime}} \quad \text { for } i=1, \ldots, s, \\
f_{j}^{(k), 2}(x, y) & =\sum_{\substack{|K| \geq 2 \\
\left|K^{\prime}\right| \geq 1}} f_{K, j}^{(k), 2} x^{K^{\prime}} y^{K^{\prime \prime}} \quad \text { for } j=1, \ldots, r,
\end{aligned}
$$

where $K=\left(K^{\prime}, K^{\prime \prime}\right) \in \mathbb{N}^{s} \times \mathbb{N}^{r}=\mathbb{N}^{n}$ and $|K|=\sum_{p=1}^{n} K_{p}$. 
Thanks to Theorem 2.4 and its proof, we know that $f_{1}$ is holomorphically linearizable via a linearization $\psi$ of the form

$$
\begin{array}{ll}
x_{i}=u_{i}+\psi_{i}^{1}(u, v) & \text { for } i=1, \ldots, s, \\
y_{j}=v_{j}+\psi_{j}^{2}(u, v) & \text { for } j=1, \ldots, r,
\end{array}
$$

where $(u, v)=\left(u_{1}, \ldots, u_{s}, v_{1}, \ldots, v_{r}\right)$ and

$$
\begin{aligned}
& \operatorname{ord}_{u}\left(\psi_{i}^{1}\right) \geq 2, \\
& \operatorname{ord}_{u}\left(\psi_{j}^{2}\right) \geq 1,
\end{aligned}
$$

that is

$$
\begin{aligned}
\psi_{i}^{1}(u, v) & =\sum_{\substack{|K| \geq 2 \\
\left|K^{\prime}\right| \geq 2}} \psi_{K, i}^{1} u^{K^{\prime}} v^{K^{\prime \prime}} \quad \text { for } i=1, \ldots, s \\
\psi_{j}^{2}(u, v) & =\sum_{\substack{|K| \geq 2 \\
\left|K^{\prime}\right| \geq 1}} \psi_{K, j}^{2} u^{K^{\prime}} v^{K^{\prime \prime}} \quad \text { for } j=1, \ldots, r .
\end{aligned}
$$

Since the linear map $\psi^{-1} \circ f_{1} \circ \psi=\operatorname{Diag}\left(\lambda_{1,1}, \ldots, \lambda_{1, s}, \mu_{1,1}, \ldots, \mu_{1, r}\right)$ commutes with $\tilde{f}_{h}=\psi^{-1} \circ f_{h} \circ \psi$ for each $h=2, \ldots, m$, and $\left(\lambda_{1,1}, \ldots, \lambda_{1, s}, \mu_{1,1}, \ldots, \mu_{1, r}\right)$ has only level $s$ resonances, it is immediate to verify that $\tilde{f}_{h}$ has the form

$$
\begin{array}{ll}
u_{i}^{\prime}=\sum_{p=1}^{s} a_{i, p}^{(h)} u_{p}+\sum_{\substack{1 \leq l \leq n \\
\lambda_{1, l}=\bar{\lambda}_{1, i}}} u_{l} \tilde{f}_{l, i}^{(h), 1}(v) & \text { for } i=1, \ldots, s, \\
v_{j}^{\prime}=f_{j}^{(h) \operatorname{lin}}(u, v)+\tilde{f}_{j}^{(h), 2}(v) & \text { for } j=1, \ldots, r .
\end{array}
$$

Moreover, since $f_{h} \circ \psi=\psi \circ \tilde{f}_{h}$, we have

$$
\begin{aligned}
& \sum_{p=1}^{s} a_{i, p}^{(h)} \sum_{\substack{|K| \geq 2 \\
\left|K^{\prime}\right| \geq 2}} \psi_{K, p}^{1} u^{K^{\prime}} v^{K^{\prime \prime}}+\sum_{\substack{|K| \geq 2 \\
\left|K^{\prime}\right| \geq 2}} f_{K, i}^{(h), 1}\left(u+\psi^{1}(u, v)\right)^{K^{\prime}}\left(v+\psi^{2}(u, v)\right)^{K^{\prime \prime}} \\
& =\sum_{\substack{1 \leq l \leq n \\
\lambda_{1, l}=\lambda_{1, i}}} u_{l} \tilde{f}_{l, i}^{(h), 1}(v) \\
& \quad+\sum_{\substack{|K| \geq 2 \\
\left|K^{\prime}\right| \geq 2}} \psi_{K, i}^{1}\left(\sum_{p=1}^{s} a_{1, p}^{(h)} u_{p}+\sum_{\substack{1 \leq l \leq n \\
\lambda_{1, l}=\bar{\lambda}_{1,1}}} u_{l} \tilde{f}_{l, 1}^{(h), 1}(v)\right)^{K_{1}} \cdots\left(\sum_{p=1}^{s} a_{s, p}^{(h)} u_{p}+\sum_{\substack{1 \leq l \leq n \\
\lambda_{1, l}=\lambda_{1, s}}} u_{l} \tilde{f}_{l, s}^{(h), 1}(v)\right)^{K_{s}} \\
& \times\left(f^{\left.(h) \operatorname{lin}(u, v)+\tilde{f}^{(h), 2}(v)\right)^{K^{\prime \prime}}}\right.
\end{aligned}
$$


for $i=1, \ldots, s$, and

(6)

$$
\begin{aligned}
& \sum_{q=1}^{r} b_{j, q}^{(h)} \sum_{\substack{|K| \geq 2 \\
\left|K^{\prime}\right| \geq 1}} \psi_{K, q}^{2} u^{K^{\prime}} v^{K^{\prime \prime}}+\sum_{p=1}^{s} c_{j, p}^{(h)} \sum_{\substack{|K| \geq 2 \\
\left|K^{\prime}\right| \geq 2}} \psi_{K, p}^{1} u^{K^{\prime}} v^{K^{\prime \prime}} \\
& +\sum_{\substack{|K| \geq 2 \\
\left|K^{\prime}\right| \geq 1}} f_{K, j}^{(h), 2}\left(u+\psi^{1}(u, v)\right)^{K^{\prime}}\left(v+\psi^{2}(u, v)\right)^{K^{\prime \prime}} \\
& =\tilde{f}_{j}^{(h), 2}(v) \\
& +\sum_{\substack{|K| \geq 2 \\
\left|K^{\prime}\right| \geq 1}} \psi_{K, i}^{2}\left(\sum_{p=1}^{s} a_{1, p}^{(h)} u_{p}+\sum_{\substack{1 \leq l \leq n \\
\lambda_{1, l}=\lambda_{1,1}}} u_{l} \tilde{f}_{l, 1}^{(h), 1}(v)\right)^{K_{1}} \ldots\left(\sum_{p=1}^{s} a_{s, p}^{(h)} u_{p}+\sum_{\substack{1 \leq l \leq n \\
\lambda_{1, l}=\lambda_{1, s}}} u_{l} \tilde{f}_{l, s}^{(h), 1}(v)\right)^{K_{s}} \\
& \times\left(f_{j}^{(h) \operatorname{lin}}(u, v)+\tilde{f}^{(h), 2}(v)\right)^{K^{\prime \prime}}
\end{aligned}
$$

for $j=1, \ldots, r$.

Now, it is not difficult to verify that there are no terms of the form $u^{K^{\prime}} v^{K^{\prime \prime}}$ with $\left|K^{\prime}\right|=1$ in the left-hand side of (5), whereas in the right-hand side terms of this form are given only by the sum of the $u_{l} \tilde{f}_{l, i}^{(h), 1}(v)$; therefore it must be

$$
\tilde{f}_{l, i}^{(h), 1}(v) \equiv 0,
$$

for all pairs $l, i$. Similarly, there are no terms of the form $u^{K^{\prime}} v^{K^{\prime \prime}}$ with $K^{\prime}=O$ in the left-hand side of (6), whereas, again, in the right-hand side terms of this form are given by $\tilde{f}_{j}^{(h), 2}(v)$ only; so

$$
\tilde{f}_{j}^{(h), 2}(v) \equiv 0 \quad \text { for } j=1, \ldots, r .
$$

This proves that $\tilde{f}_{h}$ is linear for every $h=2, \ldots, m$, that is $\psi$ is a simultaneous holomorphic linearization for $f_{1}, \ldots, f_{m}$.

The other direction is clear: if $f_{1}$ commutes with $f_{2}, \ldots, f_{m}$, and $f_{1}, \ldots, f_{m}$ are linear, then the eigenspace of $f_{1}$ relative to the eigenvalues $\mu_{1,1}, \ldots, \mu_{1, r}$ is a simultaneous osculating manifold for $f_{1}, \ldots, f_{m}$ (and $\left.f_{1}\right|_{M}, \ldots,\left.f_{m}\right|_{M}$ are linear), where $\left(\lambda_{1,1}, \ldots, \lambda_{1, s}, \mu_{1,1}, \ldots, \mu_{1, r}\right)$ is the spectrum of $f_{1}$.

Corollary 2.6. Let $f_{1}, \ldots, f_{m}$ be $m \geq 2$ germs of commuting biholomorphisms of $\mathbb{C}^{n}$, fixing the origin. Assume that $f_{1}$ has the origin as a quasi-Brjuno fixed point of order $s$, with $1 \leq s \leq n$. Then $f_{1}, \ldots, f_{m}$ are simultaneously holomorphically linearizable if and only if there exists a germ of complex manifold $M$ at $O$ of codimension $s$, invariant under $f_{h}$ for each $h=1, \ldots, m$ which is a simultaneous osculating manifold for $f_{1}, \ldots, f_{m}$ and such that $\left.f_{1}\right|_{M}, \ldots,\left.f_{m}\right|_{M}$ are simultaneously holomorphically linearizable.

As a final corollary, taking $s=n$ in Theorem 2.5, one gets the following:

Corollary 2.7. Let $f_{1}, \ldots, f_{m}$ be $m \geq 2$ germs of biholomorphisms of $\mathbb{C}^{n}$, fixing the origin. Assume that $f_{1}$ has the origin as a Brjuno fixed point, and that it commutes with $f_{h}$ for any $h=2, \ldots, m$. Then $f_{1}, \ldots, f_{m}$ are simultaneously holomorphically linearizable. 


\section{REFERENCES}

[A] M. Abate, Discrete holomorphic local dynamical systems, to appear in "Holomorphic Dynamical Systems", Eds. G. Gentili, J. Guenot, G. Patrizio, Lecture notes in Math., SpringerVerlag, Berlin, 2009, arXiv:0903.3289v1.

[B] F. Bracci, Local dynamics of holomorphic diffeomorphisms, Boll. UMI (8), 7-B (2004), 609636. MR2101654 (2005m:32034)

[Br] A. D. Brjuno, Analytic form of differential equations, Trans. Moscow Math. Soc. 25 (1971), pp. 131-288; 26 (1972), pp. 199-239. MR0377192 (51:13365)

[M] S. Marmi, An introduction to small divisors problems, I.E.P.I., Pisa, 2003.

[R] J. Raissy, Linearization of holomorphic germs with quasi-Brjuno fixed points, Math. Z. (2009), http://www.springerlink.com/content/3853667627008057/fulltext.pdf, Online First.

[S] L. Stolovitch, Family of intersecting totally real manifolds of $\left(\mathbb{C}^{n}, 0\right)$ and $C R$-singularities, preprint 2005, arXiv: math/0506052v2.

Dipartimento di Matematica, Università di Pisa, Largo Bruno Pontecorvo 5, 56127 Pisa, ITALY

E-mail address: raissy@mail.dm.unipi.it 